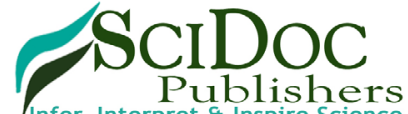

Publishers

ISSN 2572-7389

\title{
Applying Fuzzy Mathematical Model of Emotional Learning for EEG Signal Classification Between Schizophrenics and Control Participant
}

\author{
Research Article
}

Khasraghi Bj ${ }^{1 *}$, Setayeshi $\mathrm{S}^{2}$

${ }^{1}$ School of Research and Science, Islamic Azad Univesity, Tehran, Iran.

${ }^{2}$ Department of Energy Engineering and Physics, Amirkabir Univesity, Tehran, Iran.

\section{Abstract}

This paper concerns the diagnosis of schizophrenia using an electroencephalogram signals, and introduces a new framework based on the brain emotional learning model that can be applied in wide varieties of artificial intelligence applications. We propose the extended supervised version of the neuro-based computational model of an emotional learning referred as to the decay brain emotional learning based fuzzy inference system (DBELFIS). This architecture is based on fuzzy inference system, and it is build-up from the fusion algorithm based on brain emotional learning and fuzzy inference system. In this paper, we compared the proposed method with Multi-layer Perceptron (MLP), brain emotional learning (BEL) and a limbic based artificial emotional neural network (LiAENN). Substantial experimental results show that the proposed approach can effectively diagnose schizophrenia.

Keywords: Electroencephalography (EEG); Fuzzy; Schizophrenics; Amygdala; Orbitofrontal; Emotional Learning.

\section{Introduction}

Schizophrenia can be defined as a serious psychiatric disorder and falls within the scope of psychotic illnesses with a worldwide lifetime prevalence of $1 \%$. The name schizophrenia deduced from the recent scrutinization that the illness is symbolized by the disjunction or fragmentation of the different psychotic functions [1]. Patients with schizophrenia would show some types of symptoms characteristic, including disorganized speech, disturbance in thought, effects, perception and problem in social connectivity between people, the problem in inferring peoples thoughts and reacting emotionally to others [2]. The cognitive or intellectual deficit in schizophrenia that is not apparent right away requires various investigation procedures, including an effect on daily living or occupational therapists working in cognitivebehavioral therapy. The recent study established a link between certain electroencephalographic (EEG) test and patients cognitive and psychological damage [3, 4]. EEG signal demonstrates the electrical activity of the brain that analyzes useful features that can be used for the diagnosis of different diseases [5]. Patterns of EEG may correspond with the normal or impaired function of the central nervous system disease based on an empirical basis
[6]. Previous studies using EEG have investigated the emotion recognition [7], analysis of epileptic activity [8], Attention-deficit/ hyperactivity disorder (ADHD) [9], schizophrenia [10], depressive symptomatology [11], cognitive processing [12], brain damage [13] and numerous other functions. As various literature reviews show, significant problems with executive function, working memory and episode memory, reasoning, problem solving and speed of processing, in different cognitive domains have been demonstrated by people with schizophrenia $[14,15]$. However, emotional states are dynamic and may have been altered by engaging in the task, despite having complicated brain process for emotion analysis, different emotional tasks are distinguishable by measuring and describing of physiological signals [16]. Most emotions based studies have been suggested that mainly alternations in functional connectivity are seen in schizophrenia patients. There are also cognitive abnormalities with people with chronic schizophrenia [17]. Cognitive processes and emotions, both need the cortex connections involving the limbic system [18]. There have been numerous model free EEG studies between schizophrenics and other groups. Among the studies involves differentiating between the target patients population and appropriate comparison groups [19-21], denoted increased slow wave is seen significantly more



Copyright: Khasraghi $\mathbf{B J}^{\circ}$ 2017. This is an open-access article distributed under the terms of the Creative Commons Attribution License, which permits unrestricted use, distribution and reproduction in any medium, provided the original author and source are credited. 
in schizophrenia populations. Winterer et al., [22], reported increased frontally pronounced delta activity and decreased the signal power of the N100/P200 amplitude, then he concluded that schizophrenics show dysfunction of the frontal lobe. Some studies supported frontal localization of EEG abnormalities of schizophrenics [19, 23, 24]. Sponheim et al., [25] reported distinguishable ubiquity of slow wave abnormalities between schizophrenics and bipolar patients. Shagas et al., [26], compared the EEG of schizophrenics with affective disorders, personality disorders, and healthy controls. They reported a sensitivity of $50 \%$ and specificity of $90 \%$ and when comparing largely similar group, $78 \%$ sensitivity, and $85 \%$ specificity were reported. It has been proved that the magnitude of abnormal EEG was twice as great among people with schizophrenia as among affective disorder [27]. In addition, the EEG measure named Global Field Synchronization (GFS) that approximates functional connectivity of brain processes in a different EEG frequency band, was proposed; GFS analysis showed a loss of mutual dependence of memory functions in schizophrenia patients [28]. Sabeti et al., [29], several feature extraction methods, including Shannons entropy, spectral entropy, approximate entropy, Lempel-Ziv complexity, and Higuchi fractal dimension were extracted from EEG signals, then two classication methods, including LDA and Adaboost were considered, the results showed that EEG signals can be a useful tool for differentiating between the schizophrenic and control participants. Black box models [30], and white box models [30] have been applied to diagnosis issues. The well-known black box models have been applied to diagnosis disease, including neural network, linear auto regression model, non-linear auto regression model, and neuro-fuzzy inference system. However, the black box has been proven successful in modeling without having prior knowledge, but for having high accuracy, it usually needs a large number of iterations, and the model is an inconsistent truth. Regardless of its high accuracy, black box methods are usually considered as a non-transparent to the users [31, 32]. The white box physical model used to calculate an output to have physically based models with non-linear mapping that performs well for diagnosing disease and emotions based problem, and the black box neural network that has the capacity to model the network without having prior knowledge and produces an estimate of the inherent error in the physical models approximation. Fusion adaptive neuro-fuzzy inference system with the proposed methods provides more accurate output. The organization of this paper is as follows: Section 1.1, 1.2 the brain emotional learning is briefly explained, and the prior computational models are reviewed. Section 2.3 describes the proposed model. Section 3 presents the results and discussions, and Section 4 concludes the paper.

\section{Materials and Methods}

\section{Data Acquisition}

A study sample of 27 participants including 17 patient and 11 age-matched control participants (all male,18-60) recruited from consecutive admission to a major psychiatric hospital in perth, Western Australia, provided the basis for this study. All patients had been examined using a structured clinical interview the diagnostic interview for psychosis and final research diagnoses based on the DIP interview and senior consultant psychiatrist review of the clinical case notes. The patients according to DSMIV and ICD-10 criteria were diagnosed [33, 34]. The exclusion criteria for all control participant included if any of their first degree relatives had been diagnosed with schizophrenia, positive history of head injury, neurological disorder, and substance abuse or dependence at the time of testing. Variety of standard neuroleptics at the time of recording with no effort to standardize the dosages were received by patients. Each participant was seated upright with eyes closed and the experiment lasted for $2 \mathrm{~min}$. Electrophysiological data were recorded using a Neuroscan 24 Channel Synamps system, with a signal gain equal to $75 \mathrm{~K}$ (150 at the headbox). For EEG paradigms, 20 electrodes (Electrocap 1020 standard system) were recorded according to the international 10-20 system, EEG data were recorded from 20 electrodes (Fpz, Fz, Cz, Pz, C3, T3, C4, T4, Fp1, Fp2, F3, F4, F7, F8, P3, P4, T5, T6, O1, O2).

\section{Review of Brain Emotional Learning}

The neural basis of the emotional brain is represented by the limbic system (LS) theory of emotion. A group of regions in the brain containing the hippocampus, the amygdala, the thalamus, the sensory cortex and the orbitofrontal cortex, are named the limbic system. Among this structure, the amygdala plays a significant role in emotional learning and in storing emotional experiences and responses. Thalamus is the first part of the limbic system that receives emotional stimuli and is responsible for the provision of high-level information about the stimulus. LeDoux [35] announced that there are two different paths to access amygdala, one is short and fast but inaccurate and comes directly from the thalamus, the other is slow and long distance but accurate and originates from the sensory cortex. Sensory cortex distributes the incoming signal between amygdala and orbitofrontal cortex. Orbitofrontal cortex processes stimulus, learning the stimulusreinforcement association and evaluates the amygdalas response. It also evaluates the reinforcement signal and forbids the amygdala from provision of inappropriate responses [36, 37]. MacLean said interaction sensation from the world with information from the body causes emotional experience, he proposed his limbic model of emotion [38]. Lazarus announced the case for emotion involving cognition [39]. A computational model of emotional learning in the amygdala was proposed by Moren and Balkenius [36]. Brain emotional learning as a controller for heating, and air conditioning (HVAC) control system was introduced [40]. The supervised version of brain emotional learning was named FDBEL (supervised fuzzy decay brain emotional learning) was conducted for predicting Geomagnetic Index [41]. Diagnosing the complexity of the dynamic system by using a reinforcement recurrent fuzzy rule based on the brain emotional learning was designed [42]. Khashman [43] presented altered back propagation learning algorithm namely, the emotional back propagation (EmBP) learning algorithm and examined the effect of the applied emotional factors on learning and decision making potentially of the neural network.

The results showed that inserting emotional parameters improve the performance of the neural network and have high recognition rates. Khashman [44] attempted to model natural intelligence and emotions in machine learning. This approach demonstrated that emotion must be valued through simulation maps by investigating the integration of emotion at the structural level of cognitive systems by adding emotional factors on learning and decision making capabilities of the neural network. The model was named DuoNeural Network (DuoNN). The result showed that the 
DuoNN architecture, configuration, and the additional emotional information processing, obtained recognition rates with high accuracy. Kashman [43-45], assumed that the anxiety level at the beginning of a learning task is high and the confedence level is low. Brain emotional learning based fuzzy Inference system (BELFIS) integrates the idea of the previous emotional model with neuro-fuzzy inference system for predicting solar activity forecasting, it utilized adaptive networks that the number and type of membership function can be different. The results indicated that BELFIS is a reliable, nonlinear predictor model for solar activity forecasting but suffers from the curse of dimensionality and related issue; thus, it is not workable for high dimension applications [46]. Lotfi [47] modified version of brain emotional learning (BEL) applied in various control applications and proposed brain emotional learning based picture classfier (BELPIC), and the result showed the high accuracy and low time complexity for image classification. Lotfi and Akbarzadeh [48], proposed the limbic-based articial neural network (LiAENN) that models emotional situation including anxiety and confidence in the learning process, the forgetting process, the short path and inhibitory mechanisms of emotional brain. The model showed higher accuracy than other applied emotional networks such as brain emotional learning (BEL) and emotional back propagation (EmBP) based networks.

\section{Methodology}

Proposed Brain Emotional Learning Model: The proposed model is named DBELFIS (decay brain emotional learning based fuzzy inference system) was utilized to diagnose schizophrenia patients. The prominent characteristic of this approach includes fast training, methods for rules inserting, and adjusting. The fuzzy framework can explain the brain behavior [49-50]. The proposed model we fuzzfied the signal omitted from thalamus and sensory cortex using fuzzy inference system, and applied decay rate in the amygdala in order to simulate the forgetting role of amygdala, which has a biological basis [51].

Structure: The leading components of the limbic system considered for the model are the amygdala (AMY), the orbitofrontal cortex (ORB), the sensory cortex (CS), and the thalamus. The amygdala has both cortical and subcortical afferents, and it is concluded 13 interconnected nuclei, which can be divided into two main groups [52-54]. The largest (and best differentiated) and the main input structure of the amygdala complex is the basolateral nuclear group [BLA] associates conditional stimuli and unconditional representation based either on thalamic or cortical information [55]. The BLA nuclei receive input, and context from different cortical areas and the hippocampus, which appears to collectively compute possible danger and emotional salience. The other major part consists of the centromedial group [Ce-M]. Due to this fact that brain of human schizophrenia shows significant reduction of amygdala, including central and basolateral, thus the connections, and the internal parts of the amygdala are imitated by defining the BLA, and the Ce-M [56]. Patients with schizophrenia have been shown to have an actual deficit of prefrontal cortical. One of the essential elements of the prefrontal cortex is the orbitofrontal cortex [57], While the amygdala, and orbitofrontal learn about emotional and fearful stimuli, amygdala also investigates a punishment or reward value, and its main function includes monitoring, learning, reward processing, decision making, and sensory integration, while the lateral orbitofrontal cortex function is linked to the evaluation of punishers $[58,59]$. The orbitofrontal has often been subdivided into medial, lateral, posterior, and anterior sections. The thalamus is considered the gateway to the cortex. The structures of the model and connection between parts are illustrated in Figure 1. We used fusion algorithm based on amygdala and orbitofrontal emotional learning with the fuzzy inference system (see Figure 2). Data before incoming to the amygdala and orbitofrontal was fuzzfied. We simulated the function of the thalamus, and sensory cortex by applying fuzzy expert system. Due to the dynamic behavior of the model, fuzzy logic would be a suitable tool that deals with inaccurate, and incomplete data [60]. 1-Thalamus: DT has the data transfer function and passes $\mathrm{Aj}$ to sensory cortex and $\max (\mathrm{Aj})$ to amygdala, according to Eq. (1)(see Figure 1) and the graphical view of the model is showed in Figure 2.

$$
A_{j}=\max \left(A_{j}\right)-----(1)
$$

SC: Sensory cortex simulates the function of sensory cortex by applying feed forward linear neural network and disseminates $S_{k}$ between the amygdala and orbitofrontal. AMG: BLA (AB-B, LA) unit in this model averages the behavior of pyramidal excitatory neurons. The connection between amygdala weights $\left(V_{j}\right)$ and thalamus weights $\left(\mathrm{Vt}_{\mathrm{j}}\right)$ are adjusted relative to the difference

Figure 1. The Structure of FMEL Models.

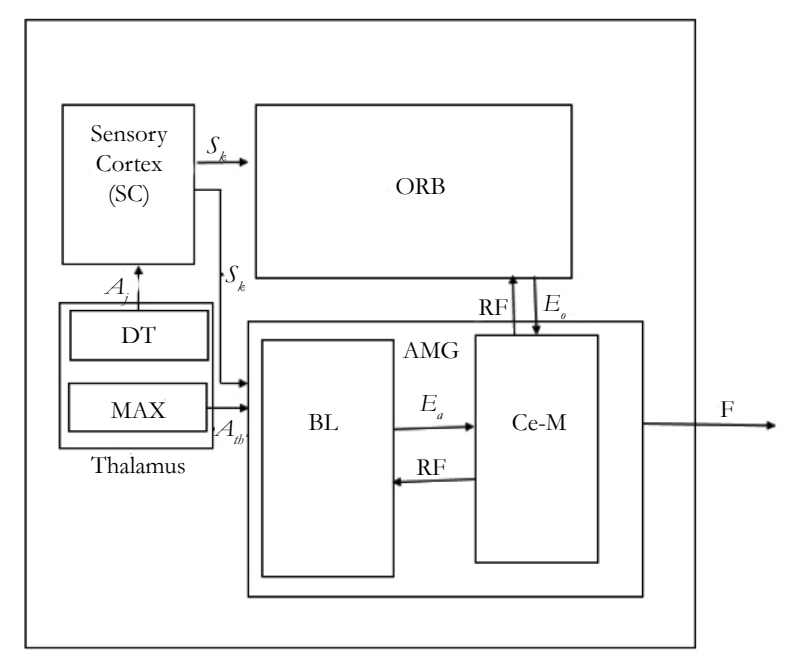


Figure 2. The Graphical Computational Model of FMEL.

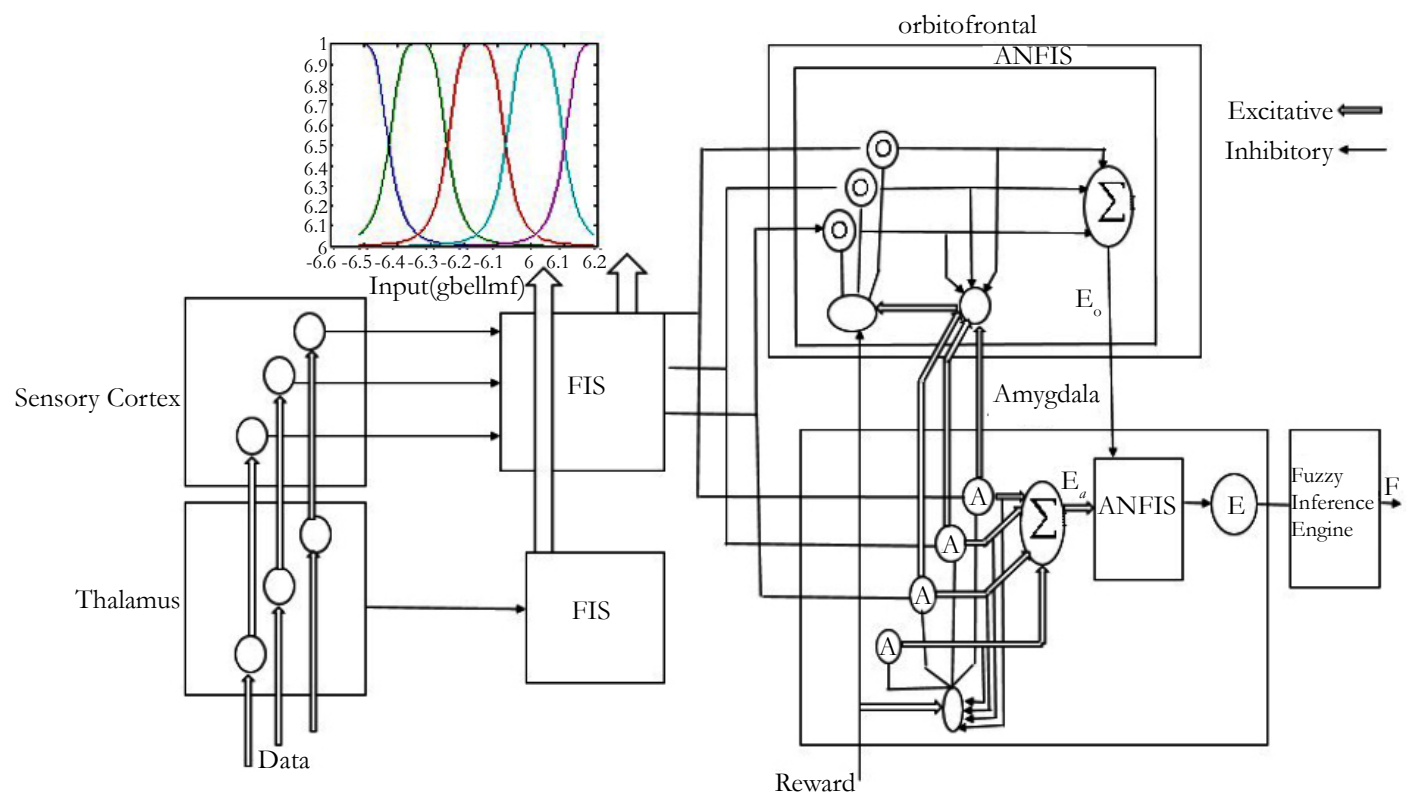

between reinforcement signal, and output of the basolateral nuclear group, $\alpha$ and $\beta$ are the learning rule, which ensure that weights do not decay to zero. The BLA passes Ea to CE-M. The connection between the Ce-M and the basolateral (BL) amygdala is excitatory in nature. The weights are adjusted based on reinforcement signal. We used decay rate $(\gamma)$ to simulate the forgetting role of amygdala theory because the neural traces of the memories are thought to disappear or decay. The left side of the eq. (2) is a bell membership function. Rules are weights (V, W) updated based on back propagation neural network algorithm. The $S_{j}$ is the $j_{\text {th }}$ fuzzy set with bell membership function and distributed between the amygdala and orbitofrontal cortex. The fusion of the bell membership function with amygdalas output, which is made by multiplying the left and right side of the equation, is shown in Eq. (2).

$E_{a}=\left(1 / 1+\left(S_{j}-W_{j}=a\right)^{2 b}\right) \cdot S_{j} \cdot V_{j}+A_{j} \cdot V t_{j}-----(2)$

$V_{j}^{i+1}=(1-\gamma) V_{j}^{i}+\alpha \max \left(\operatorname{Rew} w_{j}^{i+1}-E_{a}\right) s_{j}^{i}-----(3)$

$V t_{j}^{i+1}=(1-\gamma) V_{j}^{i}+\alpha \max \left(\operatorname{Rew}_{k}^{i+1}-E_{a}\right) A_{j}^{i}----(4)$

ORB: Orbitofrontal receives a fuzzy reinforcing signal that forbids the wrong answers of amygdala. There is a bidirectional connection between this part and the amygdala. Orbitofrontal gets $\mathrm{S}_{\mathrm{k}}$ from SC and RF from Ce-M and returns a partial response $\mathrm{E}_{\mathrm{o}}$. The $\mathrm{S}_{\mathrm{k}}$ is the $\mathrm{k}_{\mathrm{th}}$ fuzzy set with bell membership function. The ORB function is calculated by eq. (5).

$E_{o}=\left(1 /\left(1+\left(S_{j}-W_{k}\right) / a^{2 b}\right)\left(S_{k} W_{k}\right)\right.$

$\mathrm{P}_{\mathrm{o}}$ represents the expected punishment and is formulated by eq. (6).

$\mathrm{P}_{\mathrm{o}}=\mathrm{E}_{\mathrm{o}} \mathrm{W}_{\mathrm{j}+1}{ }^{-----}(6)$

The Connection weights $\mathrm{Wj}$ adjusted relative to difference between reinforcement signal and output of basolateral nuclear group according to eq. (7). $w_{j}^{i+1}=w_{j}^{j}+\beta \cdot\left(E_{o}-\operatorname{Re}_{j}^{i+1}\right) s_{j}^{i}----(7)$

$\operatorname{Rew}_{j}^{i+1}=T_{j}-E_{a}^{-----}(8)$

Ce-M: The centromedial group consists of adaptive network. The outputs of BL and OFC are crisp values. We fuzzfied the output of $\mathrm{OFC}$ and BEL as a gaussian membership function. Finally the output of the model is computed as eq. (9).

$E=\exp \left(-0.5^{*}\left(E_{a}-E_{2}\right)^{2}\right)----(9)$

\section{Results and Discussions}

The extracted feature from EEG signals should be labeled, two classes of Patient (A), and Healthy $(\mathrm{H})$ were chosen by their values. In the literature, a dimensionality reduction step has been used to classify this dataset. The defined method for the dimensionality reduction is locally linear embedding (LLE). Some advantage of LLE is to prevent the search from becoming stuck in a local minimum, and also few parameters need to be set, finding the optimal value of parameters were discussed in [61, 62]. To assess the DBELFIS method, $20 \%$ of samples were used as validation, $20 \%$ as a test and $60 \%$ as training samples. For all learning scenarios listed above, we set $\alpha, \beta$ and $\gamma 0.6,0.3$ and 0.01 . To evaluate the performance of the model, the proposed model were compared with the result of MLP, BEL, and LiAENN [48]. The number of hidden layer in MLP was defined 2. The number of rules for LiAENN, and DBELFIS are 27, and 31. All learning and testing conditions of LiAENN, and DBELFIS are the same. The predicted values by MLP, BEL, LiAENN, and DBELFIS are depicted in Table 1, it indicates that the predicted values by LiAENN, and DBELFIS are very close in the majority of points of test set, and both provide lower mse error using small number of iteration (Table 2), but DBELFIS with high specificity performs better than LiAENN as shown in Table 1. To adjust the weights we scaled all of the data between 0 and 1 . The initial weights are randomly selected between [-1, 1]. Regression analysis is a statistical process for evaluating the relationships among variables, that is widely used for prediction. Figure 3 presents the regression 
Table 1. Accuracy of Predictions.

\begin{tabular}{|c|c|c|c|}
\hline Model & Sensitivity & Specificity & Accuracy \\
\hline MLP & $93.8 \%$ & $45.5 \%$ & $74.1 \%$ \\
\hline BEL & $87.5 \%$ & $63.6 \%$ & $85.0 \%$ \\
\hline LiAENN & $100.0 \%$ & $72.7 \%$ & $88.9 \%$ \\
\hline DBELFIS & $100.0 \%$ & $81.8 \%$ & $92.6 \%$ \\
\hline
\end{tabular}

Table 2. Comparison between MLP, BEL, LiAENN, FMEL.

\begin{tabular}{|c|c|c|}
\hline Model & MSE & Structure \\
\hline MLP & $0.201 \%$ & 25epoch \\
\hline BEL & $0.1514 \%$ & 409epoch \\
\hline LiAENN & $0.01 \%$ & 44epoch \\
\hline DBELFIS & $0.008 \%$ & 19epoch \\
\hline
\end{tabular}

Figure 3. The Regression Plot in the Training Set, Test Set and Validation Set.
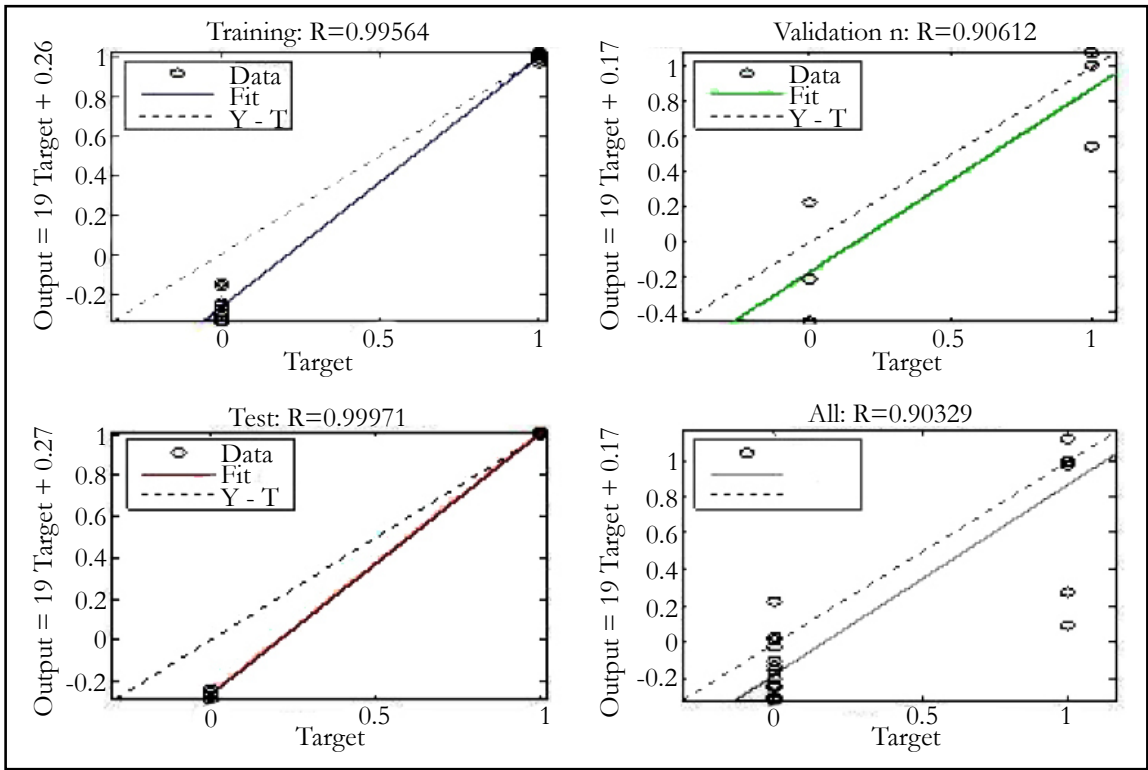

plots of the results obtained from DBELFIS. In the figures, $\mathrm{R}$ is regression value of data that represents the correlation value for the training samples $(\mathrm{R}=0.99564)$, validation samples $(\mathrm{R}=$ $0.90612)$, testing samples $(R=0.99971)$, and the fitting correlation of the whole data set on the prediction model $(\mathrm{R}=0.90329)$. This study emphasizes the advantage of merging white box and black box method to overcome the limitation of using each box including not having full control over model (black box) and their reliance to the capability of neural network to draw model (white box). This model is a novel supervised learning algorithm with a high accuracy in comparison with previous models, lower computational can achieve lower mean square error with maximum accuracy and perform well on high dimensional data. The experimental results show that proposed method forbided premature convergence problem and improved the final outputs.

\section{Conclusion}

In this research, EEG signals of 16 schizophrenic patients and 11 age-matched control participants were analyzed. A new type of brain emotional learning named DBELFIS was proposed, and applied for diagnosing schizophrenia. The DBELFIS model is a merge of the biologically-inspired model of the limbic system, and adaptive neuro-fuzzy inference system. The main modification to respect previous model is incorporating fuzzy inference system into decay brain emotional learning. The results emphasize the advantage of the fusion of fuzzy and brain emotional learning. This model is novel supervised learning algorithm with a high accuracy and its comparison with previous models can achieve lower mean square error with maximum accuracy and performs well for high dimensional data. The experimental results show that proposed method avoids premature convergence problem, and improves the final results.

\section{Acknowledgement}

The authors of this paper present a special thanks to Prof. Greg Price and Prof. Reza Khosrowabadi for their great support and advice on this project.

\section{References}

[1]. Bleuler E (1911) Dementia praecox oder Gruppe der Schizophrenien. Handbuch der psychiatrie. 442.

[2]. Spitzer RL, Williams JB (1980) Diagnostic and statistical manual of mental disorders. American Psychiatric Association.

[3]. Rissling AJ, Miyakoshi M, Sugar CA, Braff DL, Makeig S, Light GA (2014) 
Cortical substrates and functional correlates of auditory deviance processing de_cits in schizophrenia. Neuroimage: Clinical. 6: 424-37.

[4]. Subha DP, Joseph PK, Acharya UR, Lim CM (2010) EEG signal analysis: A survey. J med sys. 34(2): 195-212.

[5]. Mazhari S, Price G, Waters F, Dragovi M, Jablensky A (2011) Evidence of abnormalities in mid-latency auditory evoked responses (MLAER) in cognitive subtypes of patients with schizophrenia. Psychiatry res. 187(3): 31723.

[6]. Blanco S, Quiroga RQ, Rosso OA, Kochen S (1995) Time-frequency analysis of electroencephalogram series. Phy Review E Stat Phys Plasmas Fluids Relat Interdiscp Topics. 51(3): 2624.

[7]. Khosrowabadi R, Quek HC, Wahab A, Ang KK (2010) EEG-based emotion recognition using self-organizing map for boundary detection. In Pattern Recognition (ICPR), 20th International Conference, IEEE. 42424245 .

[8]. Adeli H, Zhou Z, Dadmehr N (2003) Analysis of EEG records in an epileptic patient using wavelet transform. J neurosci methods. 123(1): 69-87.

[9]. Clarke AR, Barry RJ, McCarthy R, Selikowitz M (1998) EEG analysis in attention-deficit/hyperactivity disorder: a comparative study of two subtypes. Psychiatry res. 81(1): 19-29.

[10]. Itil TM ( 1977) Qualitative and quantitative EEG findings in schizophrenia. Schizophr bulletin. 3(1): 61.

[11]. Rush AJ, Giles DE, Schlesser MA, Fulton CL, Weissenburger J, et al., (1986) The inventory for depressive symptomatology (IDS): preliminary findings. Psychiatry res. 18(1): 65-87.

[12]. WJ Ray, HW Cole (1985) EEG activity during cognitive processing: inuence of attentional factors. Int J Psychophysiol. 3(1): 43-48.

[13]. Watanabe K, Hayakawa F, Okumura A (1999) Neonatal EEG a powerful tool in the assessment of brain damage in preterm infants. Brain and Development. 21(6): 361-72

[14]. Barch DM, Ceaser A (2012) Cognition in schizophrenia: core psychological and neural mechanisms. Trends cogn sci. 16(1): 27-34.

[15]. MA Taylor, R Abrams (1984) Cognitive impairment in schizophrenia. Am J Psychiatry. 141(2): 196-201.

[16]. Khosrowabadi R, Heijnen M, Wahab A, Quek HC (2010) The dynamic emotion recognition system based on functional connectivity of brain regions. Intelligent Vehicles Symposium (IV), IEEE. 377-381.

[17]. Siever LJ, Davis KL (2004) The pathophysiology of schizophrenia disorders: perspectives from the spectrum. Am J Psychiatry. 161(3): 398-413.

[18]. GJ Mogenson, DL Jones, CY Yim, Mogenson GJ, Jones DL, et al., (1980) From motivation to action: functional interface between the limbic system and the motor system. Prog neurobiol. 14(2): 69-97.

[19]. Schellenberg R, Knorr W, Schindler M, Kropf S, Beyer H (1990) EEGpower spectral components of schizoaffective disorders. Schizophr res. 3(5): 357-9.

[20]. Memn EL, Floyd MT (1991) Average reference EEG lateralization in schizophrenic patients. J Neurophychiatry clin Neurosci. 3(3): 307-14.

[21]. Mientus S, Gallinat J, Wuebben Y, Pascual-Marqui RD, Mulert C, et al., (2002) Cortical hypoactivation during resting EEG in schizophrenics but not in depressives and schizotypal subjects as revealed by low resolution electromagnetic tomography (LORETA). Psychiatry Res. 116(1): 95-111.

[22]. Winterer G, Ziller M, Dorn H, Frick K, Mulert C, et al., (2000) Frontal dysfunction in schizophrenia-a new electrophysiological classifier for research and clinical applications. Eur arch psychiatry clin neurosci. 250(4): 207-14.

[23]. John ER, Prichep LS, Alper KR, Mas FG, Cancro R, et al., (1994) Quantitative electrophysiological characteristics and subtyping of schizophrenia. Biol psychiatry. 36(12): 801-26.

[24]. Omori M, Koshino Y, Murata T, Murata I, Nishio M, et al., (1995) Quantitative EEG in never-treated schizophrenic patients. Biol psychiatry. 38(5): 303-9.

[25]. Sponheim SR, Iacono WG, Clementz BA, Beiser M (1997) Season of birth and electroencephalogram power abnormalities in schizophrenia. Biol psychiatry. 41(10): 1020-7.

[26]. Shagass C, Roemer RA, Straumanis JJ, Josiassen RC (1984) Psychiatric diagnostic discriminations with combinations of quantitative EEG variables. $\mathrm{Br}$ J Psychiatry. 144(6): 581-92.

[27]. Abrams R, Taylor MA (1979) Differential EEG patterns in affective disorder and schizophrenia. Arch Gen Psychiatry. 36(12): 1355-8.

[28]. Koenig T, Lehmann D, Saito N, Kuginuki T, Kinoshita T, et al., (2001) Decreased functional connectivity of EEG theta-frequency activity in firstepisode, neuroleptic-nave patients with schizophrenia: preliminary results. Schizophrenia res. 50(1): 55-60.

[29]. Sabeti M, Katebi S, Boostani R (2009) Entropy and complexity measures for EEG signal classiffcation of schizophrenic and control participants. Artificial Intelligence in Medicine. 47(3): 263-74

[30]. Nelles O (2001) Nonlinear system identification: from classical approaches to neural networks and fuzzy models. Springer Science Business Med. 1-136.

[31]. Fraccaro P, Nicolo M, Bonetto M, Giacomini M, Weller P, et al., (2015)
Combining macula clinical signs and patient characteristics for age-related macular degeneration diagnosis: a machine learning approach. BMC ophthalmology. 15(1): 10 .

[32]. Zheng Y, Hijazi MH, Coenen F (2012) Automated disease/no disease grading of age-related macular degeneration by an image mining approach. Invest ophthalmol vis sci. 53(13): 8310-8.

[33]. Weiner EL (1996) Diagnostic and Statistical Manual of Mental Disorders: Primary Care Version. J Family Practice. 43(2): 188-90.

[34]. World Health Organnization (2004) International statistical classification of diseases and health related problems (The) ICD-10. Geneva.

[35]. LeDoux J (1998) The emotional brain. New York: Touchstone. 384

[36]. Morn J, Balkenius C (2000) A computational model of emotional learning in the amygdala. From animals to animats. Mar;6:115-24.

[37]. Morn CB (2001) Emotional learning: a computational model of the amygdala. Cyber Sys. 32(6): 611-36.

[38]. MacLean PD (1949) Psychosomatic Disease and the " Visceral Brain": recent developments bearing on the Papez theory of emotion. Psychosomatic med. 11(6): 338-53.

[39]. Lazarus RS (1982) Thoughts on the relations between emotion and cognition. Am psychol. 37(9): 1019

[40]. Sheikholeslami N, Shahmirzadi D, Semsar E, Lucas C, Yazdanpanah MJ (2006) Applying brain emotional learning algorithm for multivariable control of HVAC systems. J Intellig Fuzzy Syst. 17(1): 35-46.

[41]. Lot E, Akbarzadeh TMR (2012) Supervised brain emotional learning. Neural Networks (IJCNN), The International Joint Conference on 2012, IEEE. 1-6.

[42]. Parsapoor M, Lucas C, Setayeshi S (2008) Reinforcement-recurrent fuzzy rule based system based on brain emotional learning structure to predict the complexity dynamic system. Digital Information Management. ICDIM. Third International Conference, IEEE. 25-32.

[43]. Khashman A (2008) A modified backpropagation learning algorithm with added emotional coefficients. IEEE Trans Neural Netw. 19(11): 1896-909.

[44]. Khashman A (2010) Modeling cognitive and emotional processes: A novel neural network architecture. Neural Netw. 23(10): 1155-63.

[45]. Khashman A ( 2009) Application of an emotional neural network to facial recognition. Neural comput appli. . 18(4): 309-20.

[46]. Parsapoor M, Bilstrup U (2012) Brain emotional learning based fuzzy inference system (BELFIS) for solar activity forecasting. In Tools with Artificial Intelligence (ICTAI), IEEE 24th International Conference, IEEE. 1: 532 539.

[47]. Lotfi E (2013) Brain-Inspired Emotional Learning for Image Classification. Majlesi J Multimedia Process. 2(3): 21-26.

[48]. Lotfi E, Akbarzadeh-T MR (2014) Practical emotional neural networks. Neural Networks. 59: 61-72.

[49]. Lotfi E, Keshavarz A (2014) A simple Mathematical Fuzzy Model of Brain Emotional Learning to Predict Kp Geomagnetic Index. Intl J intellig Syst Appli Eng. 2(2): 22-5.

[50]. Reznik L, Dimitrov V, editors (1998) Fuzzy systems design: social and engineering applications. Studies in Fuzziness and soft computing. 1-85.

[51]. Gallassi R, Sambati L, Poda R, Maserati MS, Oppi F, et al., (2011) Accelerated long-term forgetting in temporal lobe epilepsy: evidence of improvement after left temporal pole lobectomy. Epilepsy Behav. 22(4): 793-5.

[52]. Aggleton JP (1985) A description of intra-amygdaloid connections in old world monkeys. Exp brain res. 57(2): 390-9.

[53]. Price JL, Russchen FT, Amaral DG (1987) The limbic region. II. The amygdaloid complex. Handbook of chemical neuroanatomy. 5(1): 279-388.

[54]. McDonald AJ (1998) Cortical pathways to the mammalian amygdala. Progneurobiol. 55(3): 257-332.

[55]. Doyre V, Schafe GE, Sigurdsson T, LeDoux JE (2003) Long-term potentiation in freely moving rats reveals asymmetries in thalamic and cortical inputs to the lateral amygdala. Eur J Neurosci. 17(12): 2703-2715.

[56]. Best B (2004) The amygdala and the emotions. Ben Best Science.

[57]. Kringelbach ML (2005) The human orbitofrontal cortex: linking reward to hedonic experience. Nat Rev Neurosci. 6(9): 691-702.

[58]. Ongr D, Price JL (2000) The organization of networks within the orbital and medial prefrontal cortex of rats, monkeys and humans. Cereb cortex. 10(3): 206-19.

[59]. Kringelbach ML, Rolls ET (2004) The functional neuroanatomy of the human orbitofrontal cortex: evidence from neuroimaging and neuropsychology. Prog neurobiol. 72(5): 341-72.

[60]. Nehdi ML, Bassuoni MT (2009) Fuzzy logic approach for estimating durability of concrete. Proceedings of the ICE-Construction Materials. 162(2): 81-92.

[61]. De Ridder D, Duin RP (2002) Locally linear embedding for classification Pattern Recognition Group: technical report series, Delft University of Technology, Delft, Netherlands. 1-15.

[62]. Kouropteva O, Okun O, Pietikinen M (2002) Selection of the optimal parameter value for the locally linear embedding algorithm. FSKD. 2. 